\title{
'Governor vessel-unblocking and mind-regulating' acupuncture therapy ameliorates cognitive dysfunction in a rat model of middle cerebral artery occlusion
}

\author{
$\mathrm{XUAN} \mathrm{SU}^{1}$, ZUQIANG WU ${ }^{2}$, FANGYONG MAI ${ }^{1}$, ZHIYONG FAN $^{3}$, \\ SHUJIA DU ${ }^{1}$, HONG QIAN ${ }^{1}$ and JINGWEN ZHU ${ }^{1}$ \\ ${ }^{1}$ Department of Rehabilitation, Guangdong Provincial Hospital of Traditional Chinese Medicine Integrated \\ with Western Medicine; ${ }^{2}$ Department of Traditional Chinese Medicine, People's Hospital of Nanhai District Foshan, \\ Foshan, Guangdong 528200; ${ }^{3}$ Department of Tuina, Guangdong Provincial Hospital of \\ Traditional Chinese Medicine, Guangzhou, Guangdong 510120, P.R. China
}

Received January 22, 2018; Accepted October 31, 2018

DOI: $10.3892 / \mathrm{ijmm} .2018 .3981$

\begin{abstract}
Acupuncture is a traditional Chinese medicinal therapy, which is used for the amelioration of cognitive dysfunction. The aim of this study was to investigate the effectiveness and relevancy mechanisms of 'governor vessel-unblocking and mind-regulating' acupuncture therapy for cognitive dysfunction in rats with ischemia. For this purpose, we used the middle cerebral artery occlusion (MCAO) method to induce cognitive dysfunction in rats. The behavioral changes in the rats were examined using the Morris water maze (MWM) test. The effects of the treatment on oxidative stress response and the function of the mitochondria in brain tissues were also assessed. The results revealed that 'governor vessel-unblocking and mind-regulating' acupuncture therapy markedly improved the cognitive ability of the rats with cognitive dysfunction. The production of pro-oxidative stress factors, including nitric oxide (NO) and inducible nitric oxide synthase (iNOS), was also blocked along with the amelioration of cognitive function, while the production of adenosine triphosphate (ATP), superoxide dismutase (SOD) and cyclooxygenase (COX) was restored. At the molecular level, the accumulation of amyloid $\beta$ $(\mathrm{A} \beta)$ in the mitochondria was suppressed by 'governor vessel-unblocking and mind-regulating' acupuncture therapy, which may be attributed to the inhibition of the function of translocase of outer mitochondrial membrane 40 (TOMM40) and translocase of inner mitochondrial membrane 17A
\end{abstract}

Correspondence to: Professor Xuan Su, Department of Rehabilitation, Guangdong Provincial Hospital of Traditional Chinese Medicine Integrated with Western Medicine, 16 South Fifth Road, Guicheng Avenue, Foshan, Guangdong 528200, P.R. China E-mail: suxuan0088@126.com

Key words: acupuncture, amyloid $\beta$, cognitive dysfunction, ischemia, mitochondrion, oxidative stress, translocase of outer mitochondrial membrane 40 , translocase of inner mitochondrial membrane 17A
(TIMM17A). On the whole, the findings of the present study confirm the effects of 'governor vessel-unblocking and mind-regulating' acupuncture therapy on cognitive dysfunction induced by brain ischemia in rats, and that the mechanisms underlying the effects of this treatment might be mediated through the inhibition of TOMM40 and TIMM17A synthesis, which can relieve mitochondrial dysfunction from the accumulation of $A \beta$.

\section{Introduction}

Cognitive impairment has been defined as a clinical state with characteristics similar to those of normal aging and mild dementia (1). The disorder is commonly diagnosed in the aged population, particularly in those suffering from Alzheimer's disease (AD), Parkinson's disease (PD), vascular dementia and ischemic stroke. Furthermore, it is the major clinical presentation in dementia with Lewy bodies (DLB) (2). Considering the aging rate of the world's population, cognitive disorders have become a critical public issue as they affect the quality of life of patients, as well as that of the caring family members $(3,4)$. Whereas a number of studies have been conducted to examine and identify the individuals who are at a high risk of suffering from cognitive dysfunction, the mechanisms of action and the effectiveness of drugs and rehabilitation treatments remain unclear. Given the poor effectiveness of modern medicine in treating cognitive dysfunction, a number of patients have begun to turn to alternative and complementary medicinal therapies for assistance.

Amongst the different alternative medicine therapies, acupuncture therapy is a commonly used treating modality in China for thousands of years against diverse disorders. The practice of acupuncture therapy encompasses a heterogeneous set of interventions, which may take action through the induction of a wide range of biological responses, either locally at the needle sites or/and distally in the peripheral nerves (5). In clinical practice in China, acupuncture has been used as an alternative therapy for patients with AD and stroke-related dementia to improve the quality of life and for 
the prevention of cognitive function decline in patients (6-9). However, controversies on the effectiveness of acupuncture still exist due to the lack of evidence on the effectiveness and mechanisms of acupuncture treatments on the nervous system. Thus, a comprehensive exploration of the mechanisms driving the effectiveness of acupuncture on cognitive dysfunction is imperative to promote the practical application of acupuncture therapy.

Emerging evidence has indicated that oxidative stress is closely related to aging and neurodegenerative diseases (10). As previously reported by Manczak et al (11), during the onset of $\mathrm{AD}$, the progression of dementia is associated with neurofibrillary tangles and the overproduction of amyloid $\beta$ (A $\beta$ ) plaques. Generally, it is accepted that the progressing accumulation of $A \beta$ will initiate a cascade of cellular changes that are lethal to the cells, including mitochondrial oxidative damage (12-14). However, the mechanisms that mediate $A \beta$ in vivo are not yet fully understood. Recent studies have indicated that translocase of outer mitochondrial membrane 40 (TOMM40) regulates the influx of $\mathrm{A} \beta$ to the mitochondria via the Tom40 outer membrane pore (15). Furthermore, according to Caselli et al (16) and Roses et al (17), TOMM40 also influences the performance of age-related memory, which indicates the potential of TOMM40 as a promising therapeutic target for cognitive dysfunction (18). Accordingly, the restoration of mitochondrial function is critical to the successful management of nerve disorders, particularly cognitive dysfunction. While the mechanisms involved are not yet fully understood, various studies have reported the improving effects of acupuncture on mitochondrial function $(19,20)$. Based on these findings, the current study aimed to investigate the mechanisms involved in the interaction between acupuncture treatment and mitochondrial function. By creating a rat model of brain ischemia induced by middle cerebral artery occlusion (MCAO), the effects of 'governor vessel-unblocking and mind-regulating' acupuncture therapy on cognitive dysfunction in the experimental rats were examined. Thereafter, the molecular mechanisms underlying the effects of acupuncture treatment on mitochondrial function were examined by focusing on the expression of TOMM40 and another translocase of mitochondrial membrane, translocase of inner mitochondrial membrane (TIMM17A). It was found that 'governor vessel-unblocking and mind-regulating' acupuncture therapy suppressed the expression of both indicators and inhibited the damage induced by $\mathrm{A} \beta$ on the mitochondria, which could resulted in the amelioration of cognitive dysfunction in rats.

\section{Materials and methods}

Chemicals and animals. Antibodies against TOMM40 (monoclonal; cat. no. 66658), TIMM17A (polyclonal; cat. no. 11189; Proteintech, Rosemont, IL, USA), amyloid precursor protein (APP; monoclonal; cat. no. ab32136), cyclooxygenase (COX, monoclonal; cat. no. ab109025), A $\beta$ (polyclonal; cat. no. ab2539) and $A \beta$ oligomer were purchased from Abcam (Cambridge, MA, USA). Antibody against GAPDH (RC-5G5) was purchased from Aksomics Inc. (Shanghai, China). Secondary HRP goat (BA1054) anti-rabbit and goat anti-mouse IgG (BA1051) antibodies were purchased from Wuhan Boster Biological Technology, Ltd. (Wuhan, China). Nimodipine
Table I. Assessment of the establishment of the MCAO model using the Longa score.

\begin{tabular}{lcc}
\hline & \multicolumn{2}{c}{ Longa score } \\
\cline { 2 - 3 } Group & Pre-surgery & Post-surgery \\
\hline Sham & $0.0 \pm 0.0$ & $0.0 \pm 0.0$ \\
MCAO & $0.0 \pm 0.0$ & $1.33 \pm 0.49^{\mathrm{a}, \mathrm{b}}$
\end{tabular}

Sham, sham-operated; MCAO, middle cerebral artery occlusion. ${ }^{\mathrm{a}} \mathrm{P}<0.001$ vs. sham group; ${ }^{\mathrm{b}} \mathrm{P}<0.001$ vs. pre-surgery.

(Nimotop; standard treatment for ischemic stroke and AD) was purchased from Qilu Pharmaceutical Co., Ltd. (Jinan, China). A total of 24 60-day-old SPF Sprague-Dawley rats (weighing $220 \pm 20 \mathrm{~g}$, female) were obtained from Guangzhou University of Chinese Medicine, Guangzhou, China, and housed at room temperature $\left(20-25^{\circ} \mathrm{C}\right)$ in a humidified chamber $(55 \pm 5 \%)$ supplemented with food and water ad libitum.

Establishment of cognitive dysfunction model using the MCAO method. All the assays using the animals were approved by the Institutional Animal Ethics Committee and Animal Care Guidelines for the Care and Use of Guangdong Provincial Hospital. In the current study, cognitive dysfunction was induced using the MCAO method. Briefly, the rats were anesthetized using $100 \mathrm{mg} / \mathrm{kg}$ ketamine plus $10 \mathrm{mg} / \mathrm{kg}$ xylazine administered via the intramuscular route. The left common carotid artery (LCCA) of the rats was exposed through a transverse neck incision, and a small incision was then made on the LCCA through which a $0.28-\mathrm{mm}$ nylon filament was introduced into the distal left internal carotid artery for the occlusion of left middle cerebral artery (LMCA), which would lead to brain infarction of its supplying region. One hour after the occlusion, the nylon filament was removed and the muscle and skin were closed in layers. The rats in the sham-operated group underwent the same surgical procedures but without the occlusion treatment. The successful establishment of the model of MCAO was assessed using the Longa score, as previously described (21) and the results are presented in Table I. The score is explained as follows: 0, no neurological deficit symptoms, activity is completely normal; 1 , mild neurological deficit, unable to fully extend the opposite front paw; 2, moderate neurological deficit, turning to the opposite side when crawling; 3 , severe neurological deficit, tilt towards the opposite side when crawling; 4, loss of consciousness, inability to crawl; 5, death. Following wound closure, the rats were housed for 10 days prior to treatment with acupuncture. The rats were deeply anesthetized with an intraperitoneal injection of pentobarbital sodium $(100 \mathrm{mg} / \mathrm{kg})$ and the brains were removed for analysis.

'Governor vessel-unblocking and mind-regulating' acupuncture therapy and animal grouping. 'Governor vessel-unblocking and mind-regulating' acupuncture therapy is based on the theory of acupoints on the $\mathrm{Du}$ channel in Traditional Chinese Medicine (TCM). In the current study, all the acupoints were recognized according to a previous publication (22) and the treatment was performed by a senior 
practitioner. Ten days after the model of MCAO was induced, the rats were fastened in a restrainer for a long period for acclimatization, which was validated by the absence of struggling. The manual twist acupunctures were then needled at the Baihui, Dazhui, Renzhong and Fengfu acupuncture points for 20 min per day for 15 days.

To assess the effects of acupuncture treatment on cognitive dysfunction, 40 rats were randomly divided into 4 groups (10 in each group) as follows: i) The sham-operated group, in which the rats underwent the same procedures as those in the surgery group only without occlusion of the arteries; ii) the MCAO group, in which the rats were subjected to MCAO for the induction of cognitive dysfunction; iii) the $\mathrm{MCAO}+$ Acupuncture group, in which the rats with cognitive dysfunction were treated with 'governor vessel-unblocking and mind-regulating' acupuncture therapy for 15 days; and iv) the $\mathrm{MCAO}+$ Nimotop group, in which the rats with cognitive dysfunction were treated with Nimotop $(20 \mathrm{mg} / \mathrm{kg}$ body weight) per day for 15 days. Upon completion of the culture, all the rats were subjected to the Morris water maze (MWM) test for the evaluation of their cognitive function. Two days after the MWM test, all the rats were sacrificed to collect cortical layer and hippocampus tissues, as well as mitochondria in brain tissues for subsequent assays.

MWM test. The MWM was used to test the learning and memorizing abilities of the rats. The assays were performed routinely as reported previously $(23,24)$ with two investigators blinded to the experimental design. The test included a 1-day probe trial and a 2-day visible platform trial. Briefly, for visible platform trail in $60 \mathrm{sec}$, the rats were allowed to swim for $60 \mathrm{sec}$ before getting to the platform for 4 times the first day and 1 time the second day. If the rats failed, the investigator would help the rats to stay on the platform for $10 \mathrm{sec}$ before another test. For probe trial in $120 \mathrm{sec}$, the time through the quadrant of the former platform position was measured.

$H \&$ EandNissl staining. The histological changes in the sections of brain tissues from the different groups were observed using H\&E staining. Briefly, the tissues were fixed in Bouin solution (4\% formaldehyde), dehydrated using alcohol and vitrified in dimethylbenzene. The samples were then embedded, sectioned and stained with hematoxylin at room temperature for $2 \mathrm{~min}$ and then with eosin for 3-5 sec. The results were studied under a microscope (CX41; Olympus Corp., Tokyo, Japan) at magnification, x400. Following H\&E staining, the nuclei in tissue were stained blue by hematoxylin and cytoplasms were stained red by eosin. The effects of acupuncture treatment on neurons in brain tissues were detected using Nissl staining following standard procedures.

Terminal-deoxynucleoitidyl transferase mediated nick-end labeling (TUNEL) staining. Cell apoptotic rates were determined using TUNEL staining. Briefly, the brain sections were permeabilized with $0.1 \%$ Triton X-100 at room temperature for $8 \mathrm{~min}$. The sections were then washed with PBS buffer prior to incubation in $3 \% \mathrm{H}_{2} \mathrm{O}_{2}$ for $10 \mathrm{~min}$ at room temperature. Following 3 5-min washes with PBS buffer, the sections were covered with TUNEL reaction solution and incubated at $37^{\circ} \mathrm{C}$ for $1 \mathrm{~h}$ in a humidified chamber in the dark. The tissues were then washed and stained with 4, 6-diamino-2-phenyl indole (DAPI) for $5 \mathrm{~min}$ at room temperature and imaged using a fluorescence microscope (FV10i; Olympus Corp.) at magnification, x400.

Immunohistochemical detection. For immunohistochemical assay, the tissue slides were placed in $60^{\circ} \mathrm{C}$ overnight prior to incubation with dimethylbenzene for dewaxing. The slides from the different groups were fixed using methanol solution with $3 \% \mathrm{H}_{2} \mathrm{O}_{2}$ and blocked with $1 \% \mathrm{BSA}$ for $30 \mathrm{~min}$ at $37^{\circ} \mathrm{C}$ and incubated with primary antibodies against TOMM40 (1:400), TIMM17A (1:200), A $\beta$ (1:500) and COX (1:500) at $4^{\circ} \mathrm{C}$ overnight. Secondary antibodies (IgG HRP; 1:3,000; cat. no. ab97051; Abcam) were added to the slides and placed at $37^{\circ} \mathrm{C}$ for $30 \mathrm{~min}$ before another 4 cycles of a PBS wash. DAB was then added to the slides and allowed to react for 3-10 min until the reaction was terminated by $\mathrm{dd}_{2} \mathrm{O}$. The slides were re-stained using hematoxylin and dehydrated. The results were recorded using a microscope (FV10i; Olympus Corp.) at magnification, $\mathrm{x} 400$.

Enzyme-linked immunosorbent assay(ELISA). The production of adenosine triphosphate (ATP), nitric oxide (NO), inducible NO synthase (iNOS) and superoxide dismutase (SOD) in the brain tissues of the different groups was measured using respective ELISA kits (Wuhan Boster Biological Technology, Ltd., China) according to the manufacturer's instructions.

Reverse transcription-quantitative PCR (RT-qPCR). Total RNA from the different samples was extracted using the RNA Purified Total RNA Extraction kit according to the manufacturer's instructions (BioTeke, Wuxi, China). Total RNA was reverse transcribed into cDNA templates using Super M-MLV reverse transcriptase (BioTeke). The final RT-PCR reaction mixture of volume $20 \mu \mathrm{l}$ consisted of $10 \mu \mathrm{l}$ of Bestar $^{\circledR}$ SUBR-Green qPCR master Mix, $0.5 \mu \mathrm{l}$ of each primer (TOMM40 forward, 5'-CTTCCTCTTCAA AGGCTCTGT-3' and reverse, 5'-ACTTATTCTTGCGGT GGTTC-3'; TIMM17A forward, 5'-CTGGCAGCAAGA AATGGA-3' and reverse, 5'-AGGCAAACCTGGTCA ACA-3'; APP forward, 5'-CCACATCGTGAT TCC TT ACC-3' and reverse, 5'-CCAGACATCGGAGTCGTCC-3'; COX forward, 5'-AGCCATTTCTACTTCGGTGTG-3' and reverse, 5'-ATTGGTGCCCTTGTTCATCT-3'; and GAPDH forward, 5'-CCTCGTCTCATAGACAAGATGGT-3' and reverse, 5'-GGGTAGAGTCATACTGGAACATG-3'), $2 \mu 1$ of the cDNA template and $7 \mu \mathrm{l}$ of Rnase free $\mathrm{H}_{2} \mathrm{O}$. The amplification parameters were set as follows: Denaturation at $94^{\circ} \mathrm{C}$ for $2 \mathrm{~min}$, followed by 40 cycles at $94^{\circ} \mathrm{C}$ for $20 \mathrm{sec}, 58^{\circ} \mathrm{C}$ for $20 \mathrm{sec}$, and $72^{\circ} \mathrm{C}$ for $20 \mathrm{sec}$. The relative mRNA expression levels were calculated with ExicyclerTM 96 (Bioneer Corporation, Daejeon, Korea) according to the expression of $2^{-\Delta \Delta \mathrm{cq}}(25)$.

Western blot analysis. Total protein product from the different groups was extracted using the Total Protein Extraction kit according to the manufacturer's instructions (Wanleibio, Beijing, China) and protein concentrations were determined using the BCA method. A total of $20 \mu \mathrm{l}$ of protein $(40 \mu \mathrm{g})$ was subjected to $10 \%$ sodium dodecylsulfate polyacrylamide gel electrophoresis (SDS-PAGE) and transferred onto polyvinyli- 

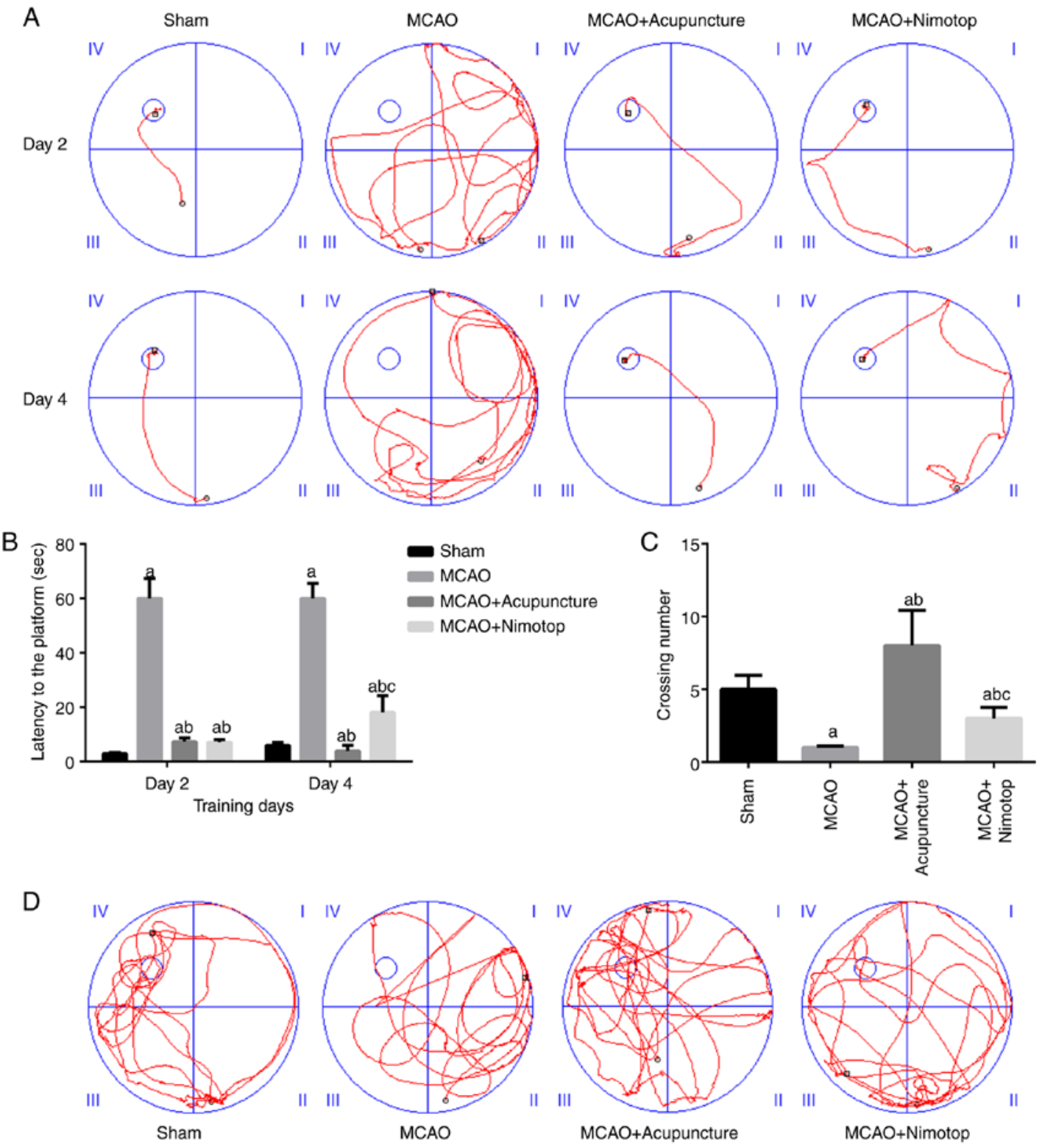

Figure 1. 'Governor vessel-unblocking and mind-regulating' acupuncture therapy improves the learning and memorizing ability of the model rats. (A) Movement track and (B) analysis results of rats in the visible platform trail. Rats in the MCAO + Acupuncture group spent less time locating the platform when compared with the MCAO group. (C) Analysis results and (D) movement track of rats in the probe trail. Rats in he MCAO + Acupuncture group spent more time and crossed more times in the quadrant of platform when compared with the MCAO group. ${ }^{\mathrm{a}} \mathrm{P}<0.05$, significantly different from the sham-operated (sham) group; ${ }^{\mathrm{b}} \mathrm{P}<0.05$, significantly different from the $\mathrm{MCAO}$ group; ${ }^{\mathrm{C}} \mathrm{P}<0.05$, significantly different from the $\mathrm{MCAO}+$ Acupuncture group. Each group is represented by 10 replicates. Error bars stand for standard deviation. MCAO, middle cerebral artery occlusion.

dene difluoride (PVDF) membranes. The membranes were then washed with TTBS for 5 min prior to incubation in skim milk powder solution for $1 \mathrm{~h}$. Primary antibodies against TOMM40 $(1: 2,000)$, TIMM17A (1:1,000), A $\beta$ (1:800), A $\beta$ oligomer (1:100), COX $(1: 800)$, and GAPDH $(1: 10,000)$ were incubated with the membranes at $4^{\circ} \mathrm{C}$ overnight and secondary HRP goat anti-rabbit antibodies $(1: 20,000)$ were incubated with the membranes for $45 \mathrm{~min}$ at $37^{\circ} \mathrm{C}$. Following additional 6 washes using TTBS, the blots were developed using Beyo ECL Plus reagent and the results were detected using the Gel Imaging System. The relative protein expression levels were calculated with Gel-Pro-Analyzer (Media Cybernetics, Rockville, MD, USA).

Flow cytometric analysis. Mitochondria were isolated from the rat hippocampal tissue using a Tissue Mitochondria Isolation kit
(\#C3606; Beyotime Institute of Biotechnology, Jiangsu, China). The isolated mitochondria of the hippocampus were stained with a cationic mitochondrion-specific dye, JC-1 (Beyotime Institute of Biotechnology) for $15 \mathrm{~min}$ at $37^{\circ} \mathrm{C}$, and washed twice with PBS. The fluorescence was analyzed using a flow cytometer (FACSCalibur; BD Biosciences, San Jose, CA, USA).

Reactive oxygen species (ROS) production in the rat hippocampal tissues was assayed using the fluorescent probe, DHE (Vigorous Biotechnology, Beijing, China). The rat hippocampal tissues obtained from the 4 experimental groups were washed, cut into sections and homogenized. The tissue homogenate was incubated with the fluorescent probe, DHE, for $20 \mathrm{~min}$ at $37^{\circ} \mathrm{C}$. Finally, the fluorescence was analyzed using a flow cytometer (FACSCalibur; BD Biosciences) according to the manufacturer's instructions. 
A

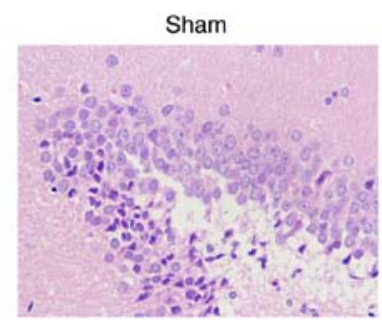

$\mathrm{MCAO}+$ Acupuncture

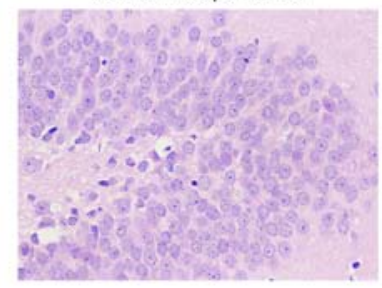

B

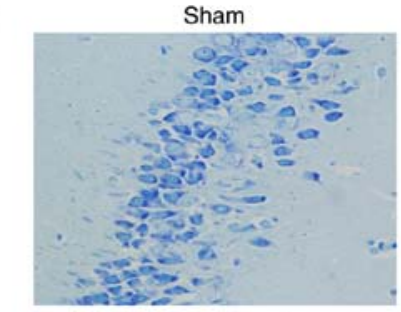

$\mathrm{MCAO}+$ Acupuncture

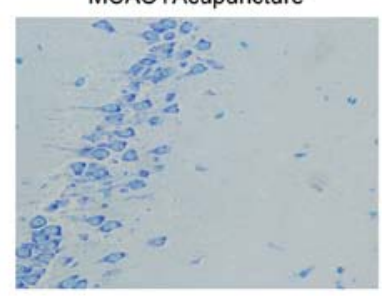

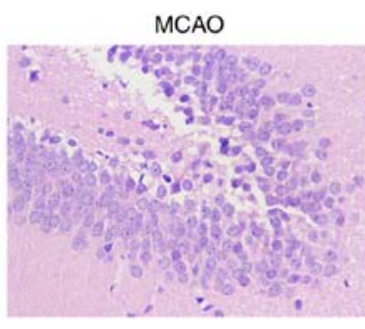

$\mathrm{MCAO}+$ Nimotop

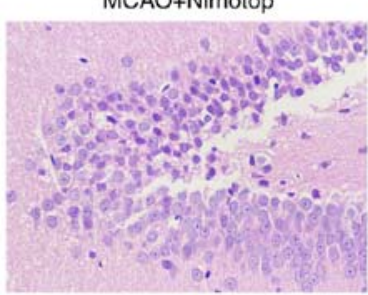

MCAO

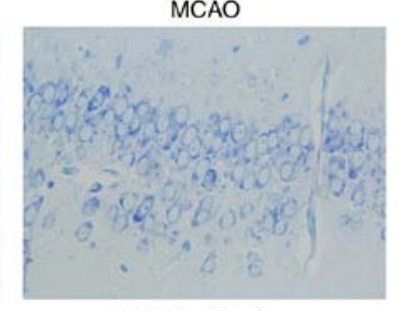

MCAO+Nimotop

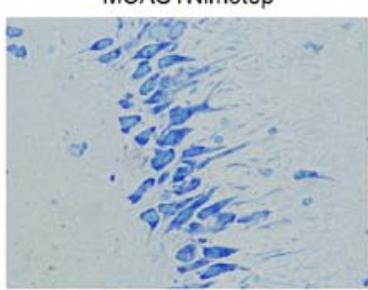

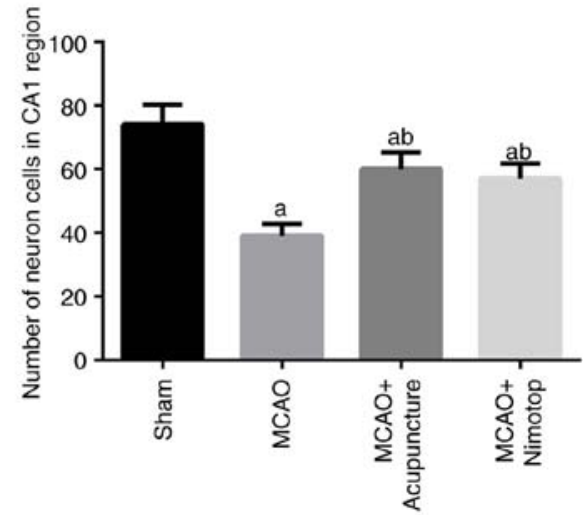

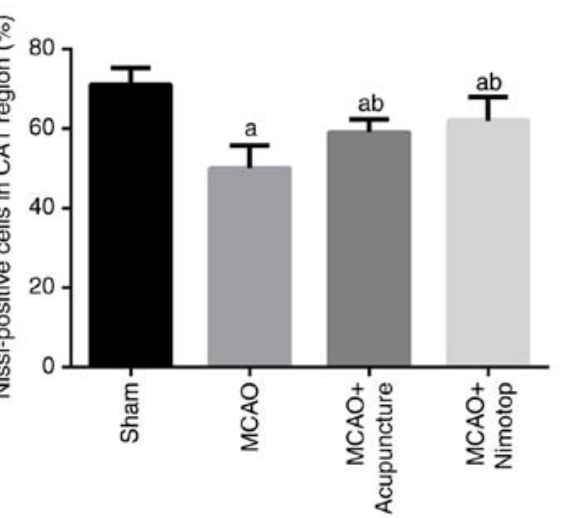

Figure 2. 'Governor vessel-unblocking and mind-regulating' acupuncture therapy alleviates the impairments in the brain tissues due to the induction of ischemia. Ischemia was associated with aging (cytoplasm stained dark pink in (A) H\&E staining (magnification, x400) and stained blue by (B) Nissl staining (magnification, $\mathrm{x} 400$ ) and the deterioration of the regular structure of the cells when compared with the sham-operated (sham) group. 'Governor vessel-unblocking and mind-regulating' acupuncture therapy alleviated the impairments induced by ischemia in the brain cells, with more cells retaining their normal shape and structure, which was comparable to the effects of Nimotop. ${ }^{\text {a }}<0.05$, significantly different from the sham-operated (sham) group; ${ }^{\text {b }}<0.05$, significantly different from the MCAO group. Each group is represented by 6 replicates. Error bars stand for standard deviation. MCAO, middle cerebral artery occlusion.

Statistical analysis. All the data are expressed as the means \pm standard deviation. For the behavior assay, each group was represented by 10 replicates. For the histological, biochemical and molecular detections, each group was represented by 6 replicates. One-way ANOVA and the post hoc LSD test was performed using the general liner model. $\mathrm{P}<0.05$ was considered to indicate a statistically significant difference. All statistical analyses were conducted using SPSS version 19.0 (IBM, Armonk, NY, USA).

\section{Results}

'Governor vessel-unblocking and mind-regulating' acupuncture therapy improves the learning and memorizing ability of rats subjected to MCAO. The results of the MWM test revealed that acupuncture treatment improved the learning and memorizing ability of the rats with cognitive dysfunction. For the visible platform trial, grouping acted as an independent factor that influenced the latency of the rats. As shown in Fig. 1A and B, the latency time of the rats in the MCAO + Acupuncture group was lower than that of the rats in the MCAO group, and the difference was statistically significant $(\mathrm{P}<0.05)$, thereby representing the restoration of the cognitive function of the rats. The results of the probe trail confirmed the conclusion of the visible platform trial, as the rats in the MCAO + Acupuncture group had a significantly higher crossing number than the rats in the other 3 groups $(\mathrm{P}<0.05)$ (Fig. 1C and D). Thus, 'governor vessel-unblocking and mind-regulating' acupuncture therapy alleviated the cognitive dysfunction of the model rats.

'Governor vessel-unblocking and mind-regulating' acupuncture therapy improves histological changes in brain tissues of MCAO rats. The histological changes in the brain tissues were detected following the induction of ischemia and acupuncture treatment. As shown in Fig. 2, the induction of cognitive dysfunction was associated with aging (cytoplasm stained dark pink by H\&E staining and stained blue by Nissl staining) and the deterioration of the regular structure of the cells when compared with the sham-operated group. Treatment with both 'governor vessel-unblocking and mind-regulating' acupuncture and Nimotop alleviated the negative effects of 


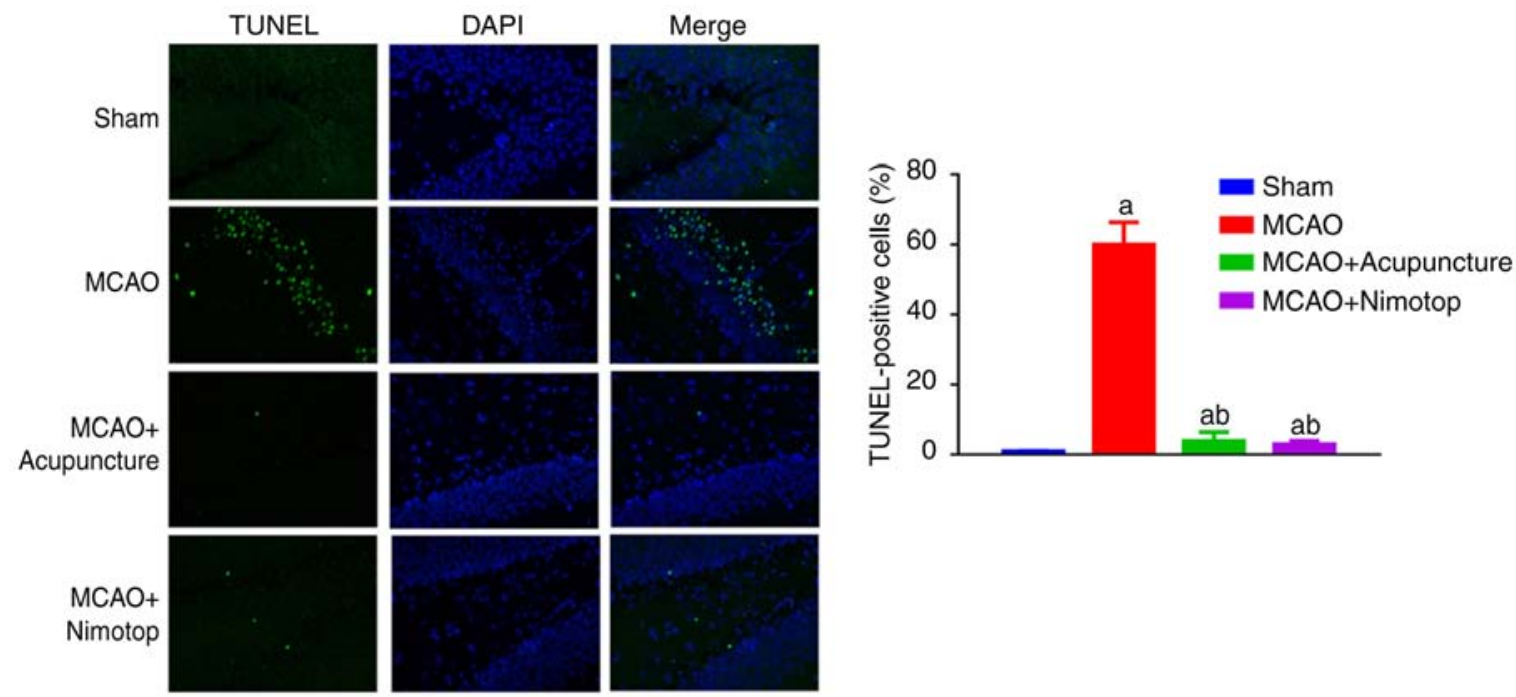

Figure 3. 'Governor vessel-unblocking and mind-regulating' acupuncture therapy suppresses cell apoptosis in brain tissues. Apoptosis was illustrated by TUNEL staining (magnification, $\mathrm{x} 400$ ). ${ }^{a} \mathrm{P}<0.05$, significantly different from the sham-operated (sham) group; ${ }^{\mathrm{b}} \mathrm{P}<0.05$, significantly different from the MCAO group. Each group is represented by 6 replicates. Error bars stand for standard deviation. MCAO, middle cerebral artery occlusion.
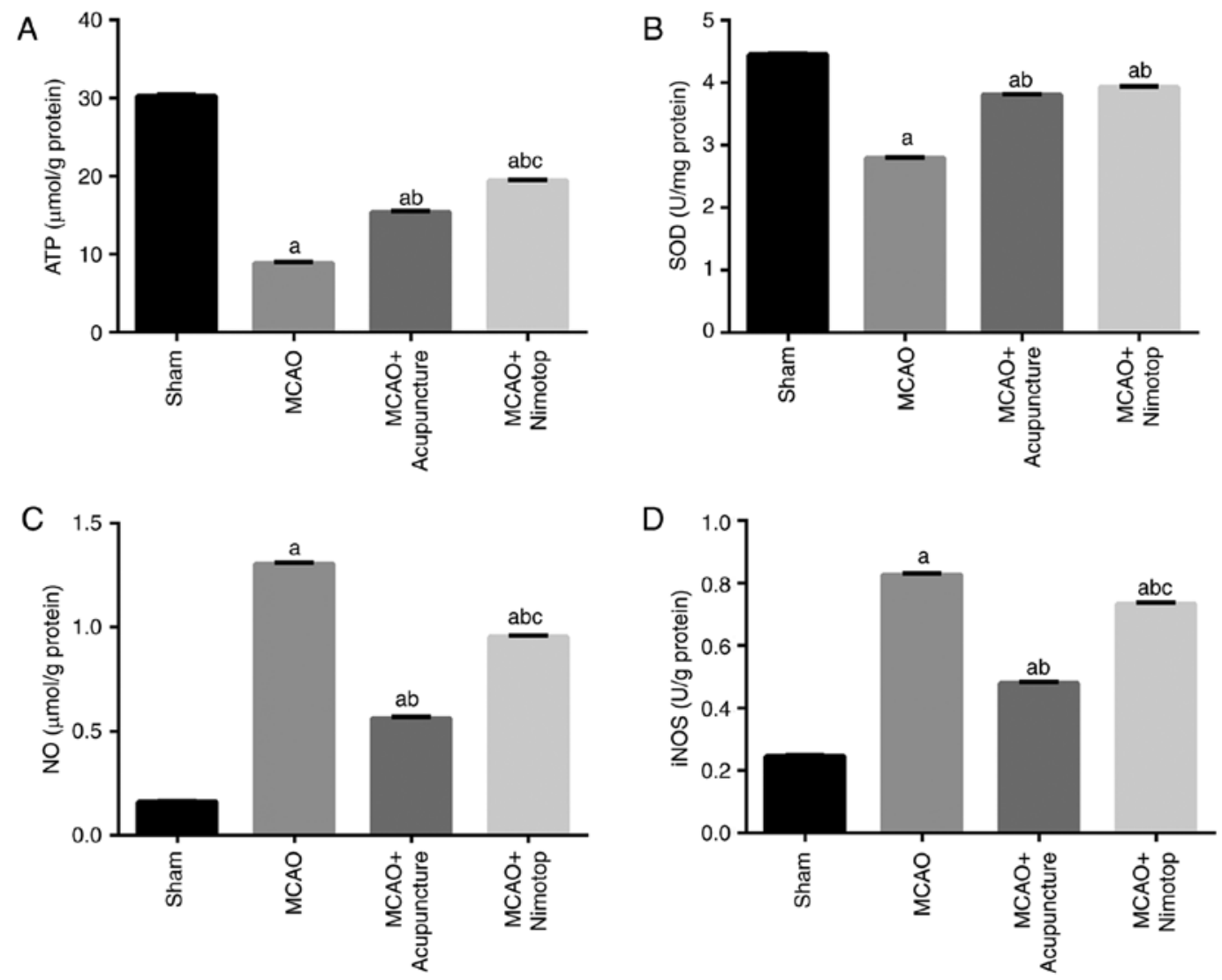

Figure 4. 'Governor vessel-unblocking and mind-regulating' acupuncture therapy suppresses oxidative stress and restores energy production in brain tissues. (A-D) Quantitative analysis results of the production of ATP, SOD, NO and iNOS. The production of ATP and SOD was inhibited by ischemia and restored by 'governor vessel-unblocking and mind-regulating' acupuncture therapy, while the production of NO and iNOS was induced by ischemia and suppressed by 'governor vessel-unblocking and mind-regulating' acupuncture therapy. ${ }^{a} \mathrm{P}<0.05$, significantly different from the sham-operated (sham) group; ${ }^{\text {b }} \mathrm{P}<0.05$, significantly different from the MCAO group; ${ }^{~} \mathrm{P}<0.05$, significantly different from the $\mathrm{MCAO}+$ Acupuncture group. Each group is represented by 10 replicates. Error bars stand for standard deviation. MCAO, middle cerebral artery occlusion; ATP, adenosine triphosphate; SOD, superoxide dismutase; NO, nitric oxide; iNOS, and inducible nitric oxide synthase.

ischemia on the brain cells, with more cells retaining their normal shape and structure (Fig. 2). In addition, as illustrated by TUNEL staining, a significantly high number of apoptotic cells (stained green) was detected in the MCAO group, and the impairments on brain tissues due to ischemia were alleviated by acupuncture treatment (Fig. 3). 

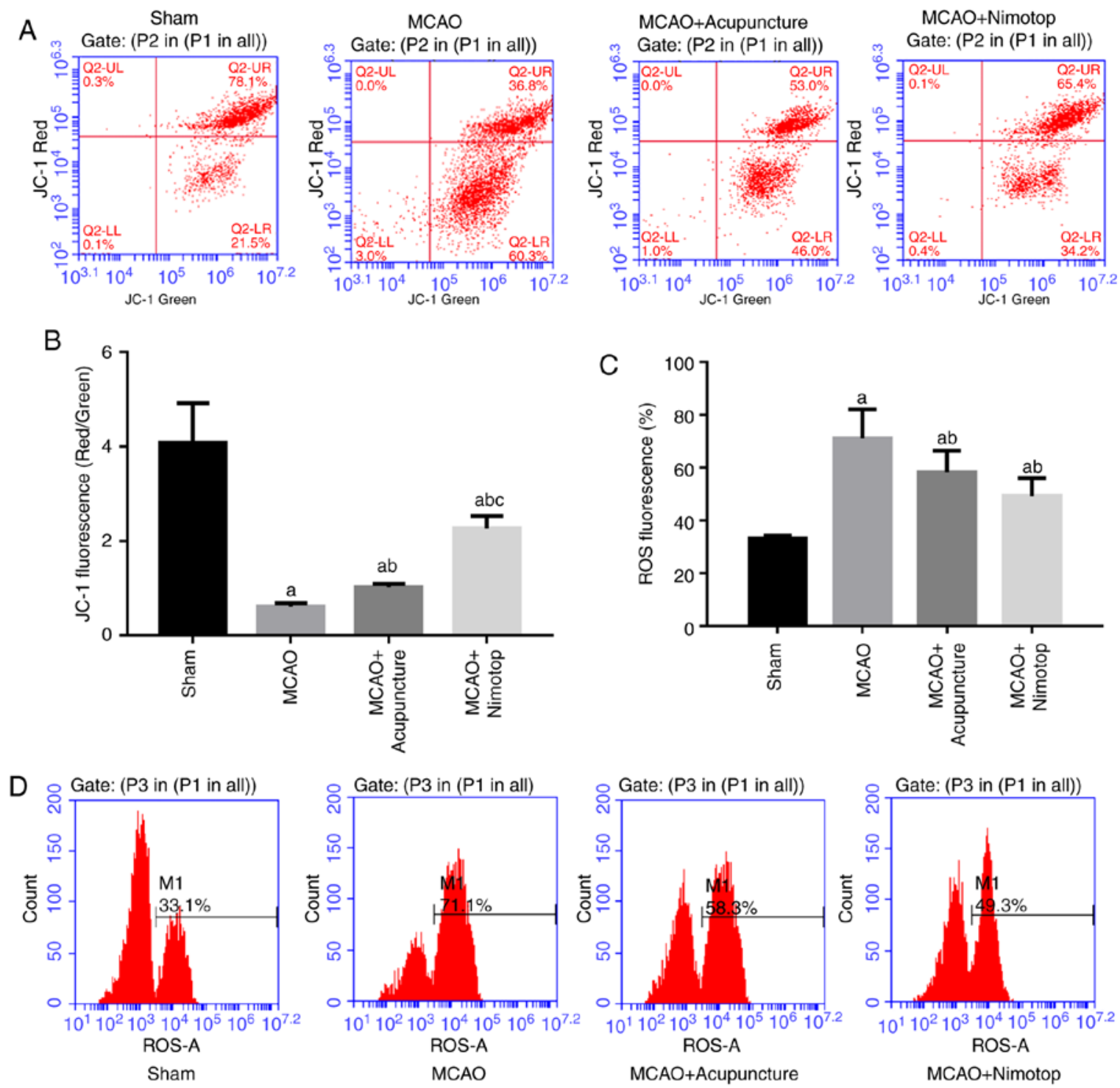

Figure 5. 'Governor vessel-unblocking and mind-regulating' acupuncture therapy improves membrane potential and suppresses oxidative stress in the brain mitochondria. (A and B) Ischemia induced membrane depolarization in the brain tissues, which was represented by a lower proportion of JC-1. 'Governor vessel-unblocking and mind-regulating' acupuncture therapy ameliorated the impairment in membrane potential in the brain mitochondria. (C and D) The level of ROS was reduced by 'governor vessel-unblocking and mind-regulating' acupuncture therapy in the MCAO + Acupuncture group. ${ }^{a} \mathrm{P}<0.05$, significantly different from the sham-operated (sham) group; ${ }^{\mathrm{b}} \mathrm{P}<0.05$, significantly different from the $\mathrm{MCAO}$ group; ${ }^{\mathrm{C}} \mathrm{P}<0.05$, significantly different from the $\mathrm{MCAO}+\mathrm{Acupuncture}$ group. Each group is represented by 10 replicates. Error bars stand for standard deviation. MCAO, middle cerebral artery occlusion; ROS, reactive oxygen species.

'Governorvessel-unblocking andmind-regulating' acupuncture therapy suppresses oxidative stress in the brain tissues of rats subjected to MCAO. Based on the results of ELISA, the level of ATP in the brain tissues of the model rats was suppressed after the induction of cognitive dysfunction; however, 'governor vessel-unblocking and mind-regulating' acupuncture therapy improved the synthesis of ATP (Fig. 4A). Moreover, the decreased production of SOD was also restored by 'governor vessel-unblocking and mind-regulating' acupuncture therapy, and the difference between the MCAO group and MCAO + Acupuncture group was statistically significant $(\mathrm{P}<0.05 ;$ Fig. 4B). The levels of factors which are upregulated during ischemia and which contribute to the pathogenesis of neurodegenerative disorders, including NO and iNOS, were suppressed by 'governor vessel-unblocking and mind-regulating' acupuncture therapy (Fig. 4C and D). Taken together, the above-mentioned results suggest the potential of 'governor vessel-unblocking and mind-regulating' acupuncture therapy to relieve brain tissues from chronic stress due to ischemia.

'Governor vessel-unblocking and mind-regulating' acupuncture therapy improves membrane potential and oxidative stress in the mitochondria in brain tissues of rats subjected to MCAO. The induction of cognitive disorders is closely associated with the dysfunction of the mitochondria in brain cells. In this study, the results from flow cytometric analysis revealed that the induction of cognitive dysfunction initiated membrane depolarization in the brain mitochondria (Fig. 5A and B): Low levels of JC-1 were measured in the MCAO group. Following 'governor vessel-unblocking and mind-regulating' acupuncture therapy, the membrane potential returned to a relatively normal level and the effect was more potent compared to treatment with Nimotop. Furthermore, the oxidative stress induced by ischemia was also alleviated by 'governor vessel-unblocking and mind-regulating' 
A

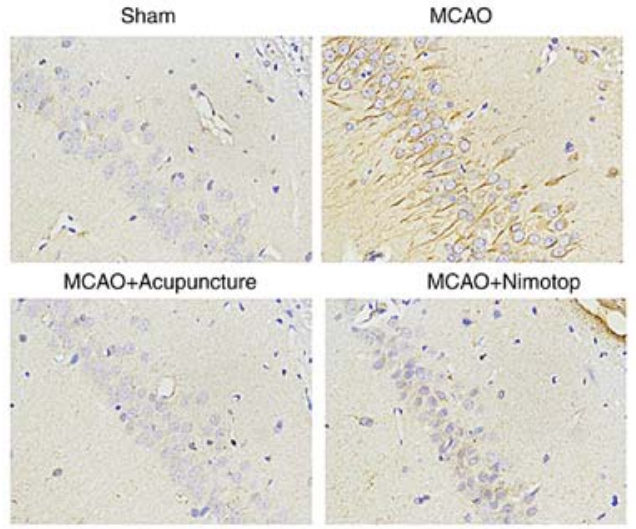

B

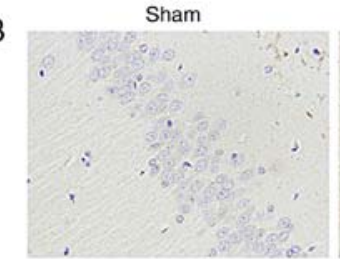

MCAO+Acupuncture

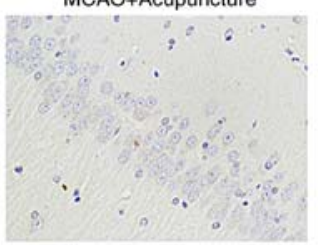

C

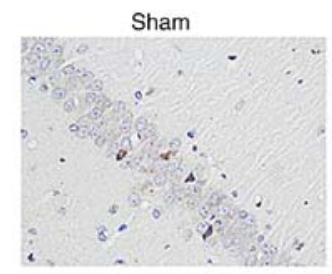

MCAO+Acupuncture

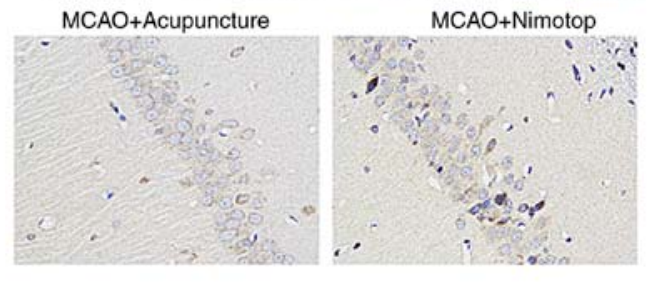

D
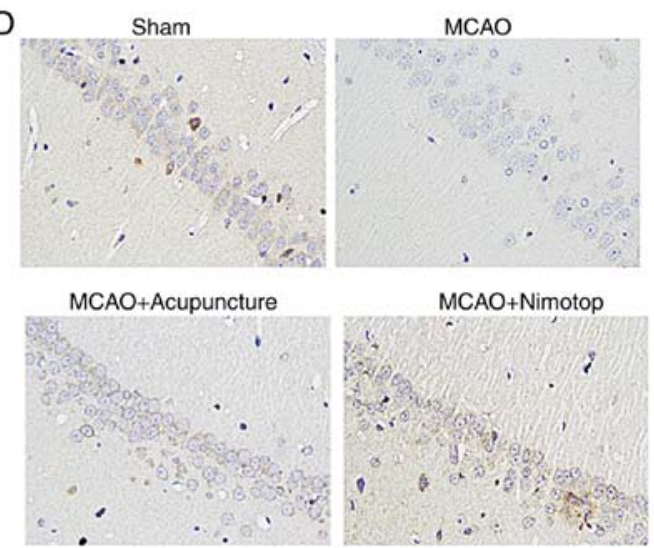

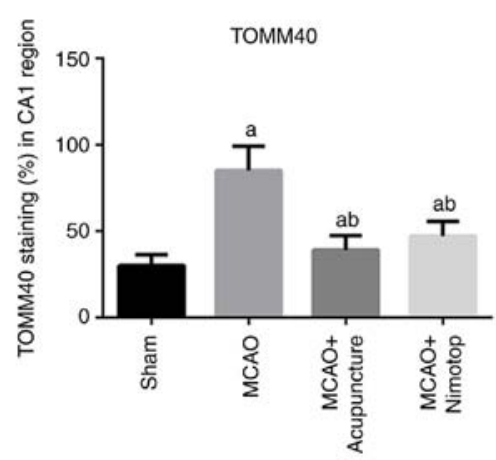

TOMM17A
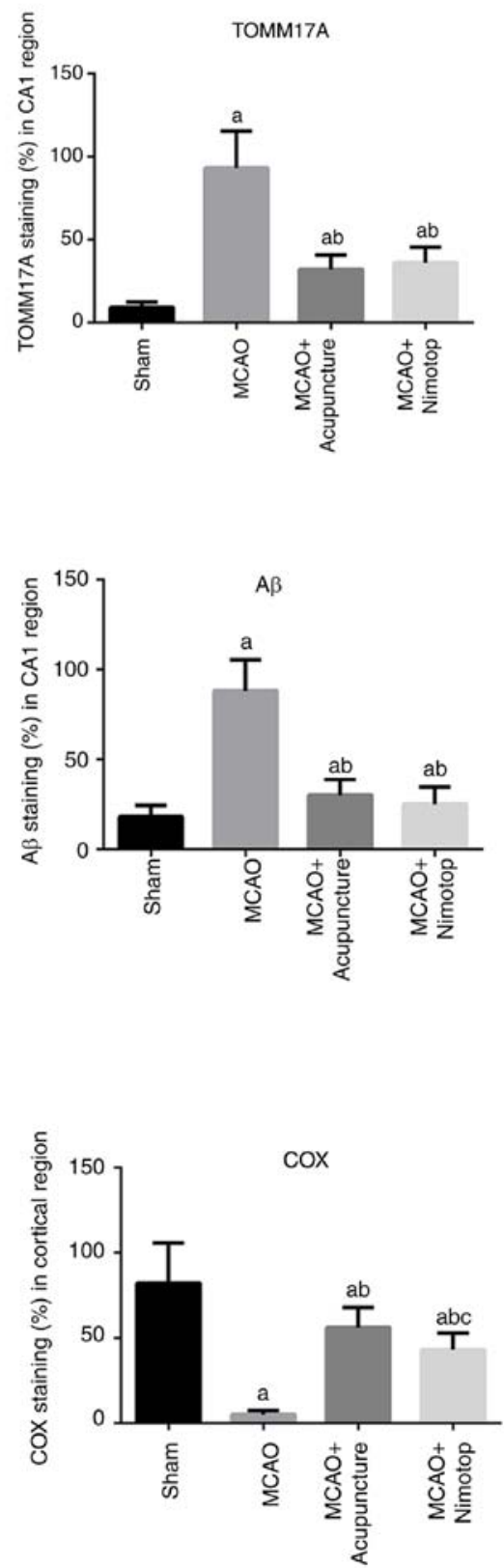

Figure 6. 'Governor vessel-unblocking and mind-regulating' acupuncture therapy inhibits the expression of TOMM40, TIMM17A and A $\beta$, while it increases the expression of COX. (A-D) Immunochemical detection (magnification, $x 400$ ) of TOMM40, TIMM17A and A $\beta$ and COX levels and quantification of these levels. ${ }^{\mathrm{a}} \mathrm{P}<0.05$, significantly different from the sham-operated (sham) group; ${ }^{\mathrm{b}} \mathrm{P}<0.05$, significantly different from the $\mathrm{MCAO}$ group; ${ }^{\mathrm{C}}<0.05$, significantly different from the MCAO + Acupuncture group. Each group is represented by 10 replicates. Error bars stand for standard deviation. MCAO, middle cerebral artery occlusion; TOMM40, translocase of outer mitochondrial membrane 40; TIMM17A, translocase of inner mitochondrial membrane $17 \mathrm{~A}$; A $\beta$, amyloid $\beta$; COX, cyclooxygenase. 
A
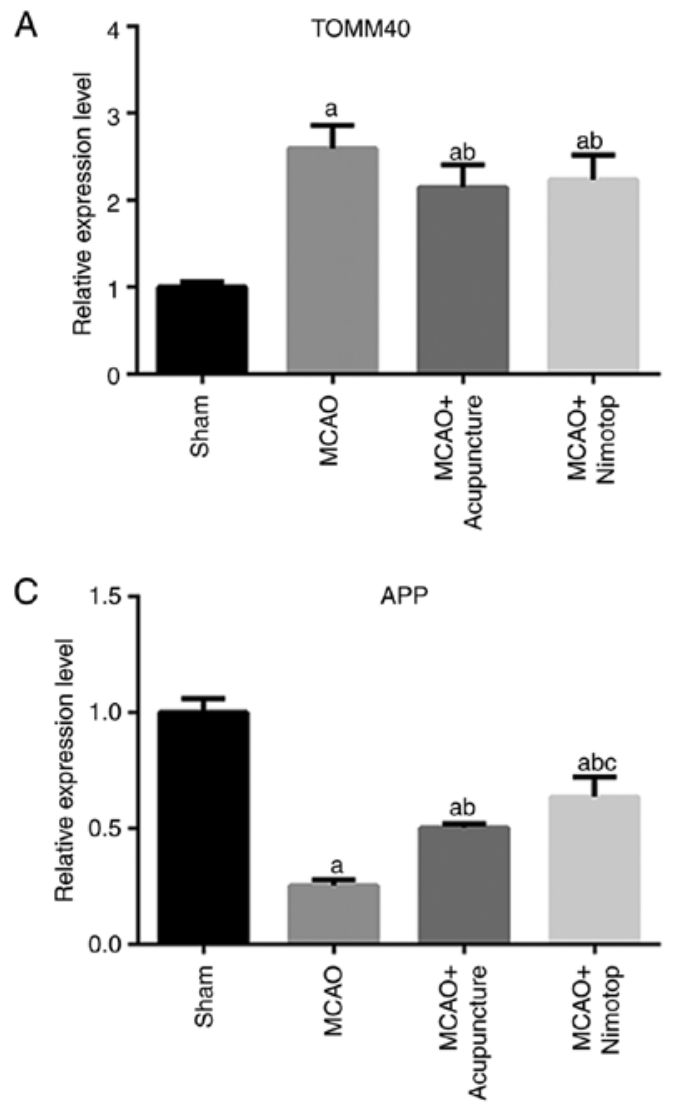

B

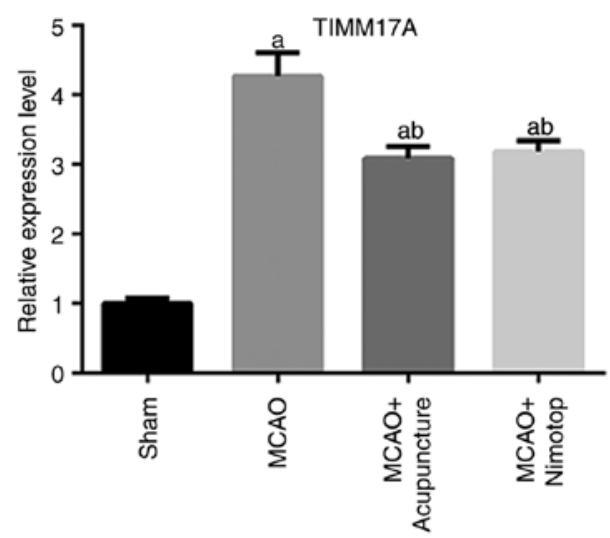

D

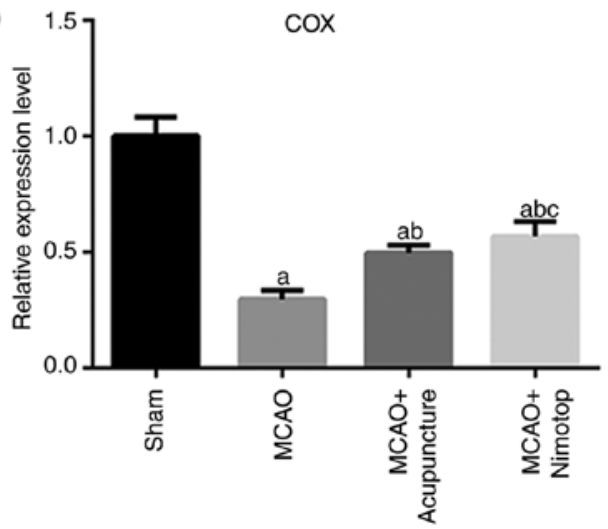

Figure 7. 'Governor vessel-unblocking and mind-regulating' acupuncture therapy exerts its effects on cognitive dysfunction by downregulating the expression of TOMM40 and TIMM17A. (A-D) Quantitative analysis results of RT-qPCR validation of TOMM40, TIMM17A, A $\beta$ and COX expression, respectively. The induction of ischemia increased the levels of TOMM40 and TIMM17A, and decreased the levels of APP and COX. 'Governor vessel-unblocking and mind-regulating' acupuncture therapy reversed the expression patterns of all the indicators. ${ }^{a} \mathrm{P}<0.05$, significantly different from the sham-operated (sham) group; ${ }^{\mathrm{b}} \mathrm{P}<0.05$, significantly different from the $\mathrm{MCAO}$ group; ${ }^{\circ} \mathrm{P}<0.05$, significantly different from the $\mathrm{MCAO}+\mathrm{Acupuncture}$ group. Each group is represented by 10 replicates. Error bars stand for standard deviation. MCAO, middle cerebral artery occlusion; TOMM40, translocase of outer mitochondrial membrane 40; TIMM17A, translocase of inner mitochondrial membrane 17A; APP, amyloid precursor protein; COX, cyclooxygenase.

acupuncture therapy, with the increased levels of ROS being inhibited in the MCAO + Acupuncture group (Fig. 5C and D).

'governor vessel-unblocking and mind-regulating' acupuncture therapy downregulates the expression of TOMM40 and TIMM17A. A previous study demonstrated that the afferent impulses induced by acupuncture are mainly transmitted by $\mathrm{A} \beta$ and A fibres (26). In the current study, the theory was taken one step further by focusing on the mediators of $A \beta$, including TOMM40 and TIMM17A. As illustrated by immunochemical detection, following the establishment of the cognitive dysfunction model, the production and distribution of TOMM40, TIMM17A and $A \beta$ were all increased in the rat brain tissues (Fig. 6A-C), while the expression of COX was downregulated (Fig.6D). As an indicator, positive cells were stained brown. With 'governor vessel-unblocking and mind-regulating' acupuncture therapy, the expression levels of these indicators were reversed. The change patterns of these indicators were synchronized with the behavioral improvement of models and function restoration of brain mitochondrion. Furthermore, the mechanisms involved in the effects of 'governor vessel-unblocking and mind-regulating' acupuncture therapy were validated by RT-qPCR (Fig. 7) and western blot analysis (Fig. 8). Apart from the quantification of the expression of TOMM40, TIMM17A and COX, the following two assays also detected the precursor of $A \beta$ and APP. It was observed that the enhanced expression of $A \beta$ led to the downregulation of APP, which was inhibited by 'governor vessel-unblocking and mind-regulating' acupuncture therapy. Given the effects of 'governor vessel-unblocking and mind-regulating' acupuncture therapy on TOMM40 and TIMM17A expression, representing its potential for modulating the influx of $A \beta$ into the mitochondria, the effects of 'governor vessel-unblocking and mind-regulating' acupuncture therapy on the transition between A $\beta$ and APP may also infer the function of 'governor vessel-unblocking and mind-regulating' acupuncture therapy to restrict the synthesis of $\mathrm{A} \beta$ as well. Moreover, the administration of acupuncture to the normal rats had no effect on the expression of COX (data not shown). Combined with the results of the behavioral tests, it can be concluded that 'governor vessel-unblocking and mind-regulating' acupuncture therapy had minial side-effects on the normal biological functions of the brain tissues of rats.

\section{Discussion}

As a traditional and potent therapeutic strategy, acupuncture has been used in the treatment of various diseases in China for centuries (27). As regards brain disorders, numerous studies have been conducted to validate its potential for improvement of not only the function of brain cells, but also the cognitive 

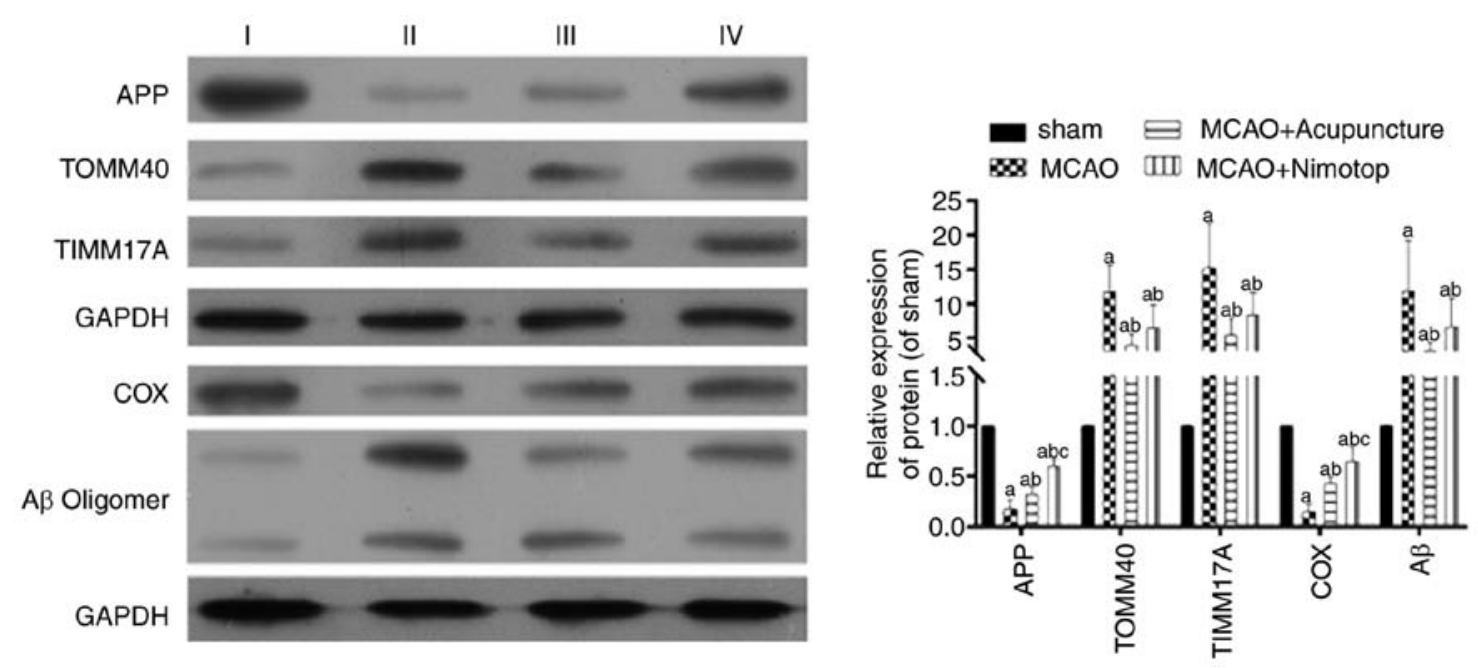

Figure 8. Representative images of western blot analysis detections of TOMM40, TIMM17A, APP, A $\beta$ and COX. The induction of ischemia increased the level of TOMM40, TIMM17A and A $\beta$ and decreased the level of APP and COX. 'Governor vessel-unblocking and mind-regulating' acupuncture therapy reversed the expression patterns of all the indicators. The lanes in the blots are labeled as follows: I, sham-operated (sham) group; II, MCAO group; III, $\mathrm{MCAO}+$ Acupuncture group; IV, MCAO + Nimotop group. ${ }^{\mathrm{a}} \mathrm{P}<0.05$, significantly different from the sham-operated (sham) group; ${ }^{\mathrm{b}} \mathrm{P}<0.05$, significantly different from the MCAO group; ${ }^{\mathrm{C}} \mathrm{P}<0.05$, significantly different from the $\mathrm{MCAO}+$ Acupuncture group. Each group is represented by 10 replicates. Error bars stand for standard deviation. MCAO, middle cerebral artery occlusion; TOMM40, translocase of outer mitochondrial membrane 40; TIMM17A, translocase of inner mitochondrial membrane 17A; APP, amyloid precursor protein; COX, cyclooxygenase.

function of patients $(1,2,28)$. Being recognized as the one of the most severe impairments of brain disorders, cognitive dysfunction severely affects the quality of life of patients with $\mathrm{AD}, \mathrm{PD}$ and vascular dementia, and poses a great threat to public health worldwide. Fortunately, the effectiveness of acupuncture treatment on cognitive dysfunction in recent years (28-30) offers an inspirable hint that the treatment can improve the prognosis of patients with brain disorders. However, due to the lack of a scientific explanation that meets the criteria of natural science and evidence-based medicine, acupuncture treatment has been classified as an alternative medicine, the effect of which is attributed to a placebo (31). Moreover, recent studies on acupuncture treatment have focused on the description of the behavioral changes of patients or model animals instead of exploring the underlying molecular mechanisms associated with the treatment $(29,30)$. To promote the application of the therapy in clinical treatment and its acceptance by modern medicine, comprehensive investigations on the pathways through which acupuncture treatment might take its action is in demand. Therefore, in the current study, the effect of 'governor vessel-unblocking and mind-regulating' acupuncture therapy on the expression levels of indicators related to mitochondrial function in brain tissues were examined. The results revealed that the treatment inhibited the expression levels of TOMM40 and TIMM17A, as well as the accumulation of $\mathrm{A} \beta$ in the brain mitochondria.

In the theory of TCM, cognitive dysfunction results from the deficiency and/or dysfunction of 'Yang Qi', the traveling of which in the human body depends on the clear passage in the 'Du' channel (32). Therefore, therapies that can improve the function of the 'Du' channel may contribute to the amelioration of cognitive dysfunction. Accordingly, 'governor vessel-unblocking and mind-regulating' acupuncture therapy was developed and employed in the current study for cognitive improvement in a rat model. Theoretically, the method stimulates the points belonging to the 'Du' channel in the brain using acupuncture, opening up the 'Du' channel and restoring neural function.
The effects of 'governor vessel-unblocking and mind-regulating' acupuncture therapy were firstly validated in a behavioral test. The MWM is a powerful tool for assessing spatial learning and memory in rats. In the current study, rats in the MCAO group exhibited cognitive impairments in the MWM test, i.e., they reached the platform with a longer latency and remained in the quadrant where the platform had been previously located with less time, indicating the deficiency in learning and memorizing ability. Following acupuncture treatment, the rats in the MCAO + Acupuncture group exhibited an improvement in the acquisition in the visible platform trial and retention in the probe trial: They reached the platform with lower latency and crossed over former platform position more frequently. Apart from the MWM test, the effects of 'governor vessel-unblocking and mind-regulating' acupuncture therapy were also verified by $H \& E$ staining, in which the brain cells of rats in the $\mathrm{MCAO}+$ Acupuncture group retained their normal structure compared with those of the rats in the MCAO group. It was more inspirable to record that the rats in MCAO + Acupuncture group even performed better in the MWM test than those in the $\mathrm{MCAO}+$ Nimotop group, indicating a potent treatment effect of 'governor vessel-unblocking and mind-regulating' acupuncture therapy for cognitive dysfunction.

In addition to the ameliorating effects on the brain cell structure and brainfunction of model rats, 'governor vessel-unblocking and mind-regulating' acupuncture therapy suppressed oxidative stress in the brain tissues of ischemic rats. Following treatment with 'governor vessel-unblocking and mind-regulating' acupuncture, the production of ATP and SOD was augmented, while the production of NO and iNOS was decreased. Oxidative stress plays a vital role in neuronal damage and in cognitive deficits in the elderly (33) and is frequently recorded along with symptoms in patients with $\mathrm{AD}$ and $\mathrm{PD}$ (34). As the major source of free radicals in cells, the mitochondria are easily affected by the oxidative stress associated with brain disorders $(35,36)$. In the current study, the membrane potential of the mitochondria 
was depolarized following the induction of cognitive dysfunction. Furthermore, the production of ROS in the mitochondria was increased, confirming the functional dysregulation of the mitochondria induced by ischemia. The abnormality of mitochondrial function was accompanied by impairments in energy production (less ATP production) in the brain tissues of the model rats, which was particularly important in view of the significant mental retardation characteristic of patients with AD, PD and vascular dementia (29). Following treatment with 'governor vessel-unblocking and mind-regulating' acupuncture, the membrane potential and ROS production in the mitochondria were restored to their normal levels. Such a modulating effect of acupuncture on mitochondrial function is vital for targeted interventions on the mitochondria in delaying AD progression in elderly individuals $(11,37,38)$. The possible interaction between acupuncture treatments and mitochondrial functions was also reported by Lu. They showed that acupuncture can induce afferent impulses transmitted by A $\beta$ fibres (39).

$\mathrm{A} \beta$ is generated by the abnormal processing of APP, which constitutes a major component of neurotic plaques or amyloid deposits found in brains affected by AD and PD $(40,41)$. The accumulation of $A \beta$ in the mitochondria suppresses the activity of COX and impairs mitochondrial metabolism (11). The results were verified in the current study by detections at molecular levels. The expression of COX was decreased both at the mRNA and protein levels following the induction of cognitive dysfunction, whereas the expression of $A \beta$ increased. We also demonstrated the mechanisms involved in the effects of acupuncture on cognitive dysfunction. It has been hypothesized that the mitochondria exert neurotoxicity by allowing the influx of $A \beta$ to cells via the Tom40 import pore (18). The pore is governed by TOMM40 and is essential for mitochondrial survival $(42,43)$. Consequently, the expression of TOMM40 was detected in the current study. In addition, for the exploration of novel biomarkers associated with the dysregulation of $\mathrm{A} \beta$, the expression of TIMM17A was also primarily assessed. It was found that both indicators were upregulated in the rats with cognitive dysfunction. Following treatment with 'governor vessel-unblocking and mind-regulating' acupuncture, the levels of TOMM40 and TIMM17A were suppressed with the decrease in the A $\beta$ levels. Previous studies have indicated that TOMM40 influences the $\mathrm{A} \beta$ influx in an ApoE-dependent manner (44-46). However, no study to date has yet reported the association between $A \beta$ and TIMM17A, at least to the best of our knowledge. The indicator is only proven to be associated with the adverse pathological and clinical outcomes in human breast cancer (47). Based the results of the current study, TIMM17A may also participate in the effects of acupuncture on cognitive dysfunction, although the detail mechanisms warrant further investigation.

Conclusively, in this study, we demonstrated that treatment with 'governor vessel-unblocking and mind-regulating' acupuncture contributed to the amelioration of cognitive dysfunction in rats subjected to MCAO. Following the administration of acupuncture, the accumulation of $A \beta$ in the brain mitochondria was inhibited. The process was mediated through the suppression on TOMM40 and TIMM17A by acupuncture stimulation. The findings outlined in this study provide insight into the molecular mechanisms associated with the treatment of acupuncture. 'governor vessel-unblocking and mind-regulating' acupuncture therapy not only influenced the behavior of the rats subjected to MCAO, but also modulated the signaling pathway involved in the pathogenesis of cognitive dysfunction. Our results may prove to be an inspiration for the scientific explanation of effects of acupuncture treatment. Further comprehensive studies are required to promote the application of this treatment modality in clinical practice in the future.

\section{Acknowledgements}

Not applicable.

\section{Funding}

The present study was supported by the Guangdong Province Enterprise Technology R\&D and appreciation transformation special fund project plan (2013B021800211).

\section{Availability of data and materials}

All data generated or analyzed during this study are included in this published article or are available from the corresponding author on reasonable request.

\section{Authors' contributions}

XS designed the research, collected the data, and wrote the draft. ZW collected and analyzed the data. FM collected the data. ZF performed the data analysis. SD designed the experiment and revised the draft. HQ designed the research. JZ wrote the draft and designed the research. All authors have read and approved the final manuscript.

\section{Ethics approval and consent to participate}

All the assays using the animals were approved by the Institutional Animal Ethics Committee and Animal Care Guidelines for the Care and Use of Guangdong Provincial Hospital.

\section{Patient consent for publication}

Not applicable.

\section{Competing interests}

The authors declare that they have no competing interests.

\section{References}

1. Lu X, Hongcai S, Jiaying W, Jing $\mathrm{H}$ and Jun $\mathrm{X}$ : Assessing the quality of reports about randomized controlled trials of acupuncture treatment on mild cognitive impairment. PLoS One 6: e16922, 2011

2. Leung MC, Yip KK, Ho YS, Siu FK, Li WC and Garner B: Mechanisms underlying the effect of acupuncture on cognitive improvement: A systematic review of animal studies. J Neuroimmune Pharmacol 9: 492-507, 2014.

3. Aggarwal NT, Tripathi M, Dodge HH, Alladi S and Anstey KJ: Trends in Alzheimer's disease and dementia in the Asian-pacific region. Int J Alzheimers Dis 2012: 171327, 2012.

4. World Health Organization and Alzheimer's Disease International: Dementia: A public health priority. World Health Organization, Geneva, 2012.

5. Rabinstein AA and Shulman LM: Acupuncture in clinical neurology. Neurologist 9: 137-148, 2003. 
6. Yu J, Zhang X, Liu C, Meng Y and Han J: Effect of acupuncture treatment on vascular dementia. Neurol Res 28: 97-103, 2006.

7. Chou P, Chu H and Lin JG: Effects of electroacupuncture treatment on impaired cognition and quality of life in Taiwanese stroke patients. J Altern Complement Med 15: 1067-1073, 2009.

8. Shen PF, Kong L, Ni LW, Guo HL, Yang S, Zhang LL, Zhang ZL, Guo JK, Xiong J, Zhen Z and Shi XM: Acupuncture intervention in ischemic stroke: A randomized controlled prospective study. Am J Chin Med 40: 685-693, 2012.

9. Shi GX, Liu CZ, Li QQ, Zhu H and Wang LP: Influence of acupuncture on cognitive function and markers of oxidative DNA damage in patients with vascular dementia. J Traditional Chin Med 32: 199-202, 2012.

10. Wang J, Markesbery WR and Lovell MA: Increased oxidative damage in nuclear and mitochondrial DNA in mild cognitive impairment. J Neurochem 96: 825-832, 2006.

11. Manczak M, Anekonda TS, Henson E, Park BS, Quinn J and Reddy PH: Mitochondria are a direct site of $A \beta$ accumulation in Alzheimer's disease neurons: Implications for free radical generation and oxidative damage in disease progression. Hum Mol Genetics 15: 1437-1449, 2006.

12. Pappolla MA, Omar RA, Kim KS and Robakis NK: Immunohistochemical evidence of oxidative [corrected] stress in Alzheimer's disease. Am J Pathol 140: 621-628, 1992.

13. Bozner P, Grishko V, LeDoux SP, Wilson GL, Chyan YC and Pappolla MA: The amyloid beta protein induces oxidative damage of mitochondrial DNA. J Neuropathol Exp Neurol 56 1356-1362, 1997.

14. Pappolla MA, Chyan YJ, Omar RA, Hsiao K, Perry G, Smith MA and Bozner P: Evidence of oxidative stress and in vivo neurotoxicity of beta-amyloid in a transgenic mouse model of Alzheimer's disease: A chronic oxidative paradigm for testing antioxidant therapies in vivo. Am J Pathol 152: 871-877, 1998.

15. Yu CE, Seltman H, Peskind ER, Galloway N, Zhou PX Rosenthal E, Wijsman EM, Tsuang DW, Devlin B and Schellenberg GD: Comprehensive analysis of APOE and selected proximate markers for late-onset Alzheimer's disease: Patterns of linkage disequilibrium and disease/marker association. Genomics 89: 655-665, 2007.

16. Caselli RJ, Dueck AC, Huentelman MJ, Lutz MW, Saunders AM, Reiman EM and Roses AD: Longitudinal modeling of cognitive aging and the TOMM40 effect. Alzheimers Dement 8: 490-495, 2012.

17. Roses AD, Lutz MW, Crenshaw DG, Grossman I, Saunders AM and Gottschalk WK: TOMM40 and APOE: Requirements for replication studies of association with age of disease onset and enrichment of a clinical trial. Alzheimers Dement 9: 132-136, 2013

18. Ferencz B, Karlsson S and Kalpouzos G: Promising genetic biomarkers of preclinical Alzheimer's disease: The influence of APOE and TOMM40 on brain integrity. Int J Alzheimers Dis 2012: 421452, 2012

19. Zhang X, Wu B, Nie K, Jia Y and Yu J: Effects of acupuncture on declined cerebral blood flow, impaired mitochondrial respiratory function and oxidative stress in multi-infarct dementia rats. Neurochem Int 65: 23-29, 2014

20. Peng J, Zeng F, He YH, Tang Y, Yin HY and Yu SG: Observation on the protective effect of electroacupuncture on hippocampal neuronal mitochondria in SAMP 8 mice. Acupuncture Res 32: 364 2007 (In Chinese).

21. Li RQ, Wan MY, Shi J, Wang HL, Liu FL, Liu CM, Huang J, Liu RC, Ma L and Feng XD: Catgut implantation at acupoints increases the expression of glutamate aspartate transporter and glial glutamate transporter-1 in the brain of rats with spasticity after stroke. Neural Regen Res 13: 1013-1018, 2018.

22. Hua XB: The development of the rat acupuncture point. Exper Anim Anim Exper 1: 1-6, 1991.

23. Chu Q, Yu J and Han J: Improvement of acupuncture on cognitive function in senescence accelerated mouse P8. Chin J Behav Med Sci 14: 964-965, 2005

24. Liu CZ, Yu JC, Cheng HY, Jiang ZG, Li T, Zhang XZ, Zhang LL and Han JX: Spatial memory performance and hippocampal neuron number in osteoporotic SAMP6 mice. Exp Neurol 201 452-460, 2006

25. Livak KJ and Schmittgen TD: Analysis of relative gene expression data using real-time quantitative PCR and the 2(-Delta Delta C(T)) method. Methods 25: 402-408, 2001.

26. Kim SA, Lee BH, Bae JH, Kim KJ, Steffensen SC, Ryu YH, Leem JW, Yang CH and Kim HY: Peripheral afferent mechanisms underlying acupuncture inhibition of cocaine behavioral effects in rats. PLoS One 8: e81018, 2013.
27. Patil S, Sen S, Bral M, Reddy S, Bradley KK, Cornett EM, Fox CJ and Kaye AD: The role of acupuncture in pain management. Curr Pain Headache Rep 20: 22, 2016.

28. Johnston MF, Yang C, Hui KK, Xiao B, Li XS and Rusiewicz A: Acupuncture for chemotherapy-associated cognitive dysfunction: A hypothesis-generating literature review to inform clinical advice. Integr Cancer Ther 6: 36-41, 2007.

29. Yu J, Liu C, Zhang X and Han J: Acupuncture improved cognitive impairment caused by multi-infarct dementia in rats. Physiol Behav 86: 434-441, 2005.

30. Liu CZ, Yu JC, Zhang XZ, Fu WW, Wang T and Han JX: Acupuncture prevents cognitive deficits and oxidative stress in cerebral multi-infarction rats. Neurosci Lett 393: 45-50, 2006.

31. Madsen MV, Gøtzsche PC and Hróbjartsson A: Acupuncture treatment for pain: Systematic review of randomised clinical trials with acupuncture, placebo acupuncture, and no acupuncture groups. BMJ 338: a3115, 2009.

32. Zeng $\mathrm{F}$ and $\mathrm{Yu}$ SG: The mechanism involved in the treatment of senile dementia via regulation fo Du Channel. J Sichuan Tradit Chin Med 22: 19-21, 2004 (In Chinese).

33. Cantuti-Castelvetri I, Shukitt-Hale B and Joseph JA: Neurobehavioral aspects of antioxidants in aging. Int $J$ Dev Neurosci 18: 367-381, 2000.

34. Ryglewicz D, Rodo M, Kunicki PK, Bednarska-Makaruk M, Graban A, Lojkowska W and Wehr H: Plasma antioxidant activity and vascular dementia. J Neurol Sci 203: 195-197, 2002.

35. Wallace DC: Mitochondrial genetics: A paradigm for aging and degenerative diseases? Science 256: 628-632, 1992.

36. Wallace DC: Mitochondrial diseases in man and mouse. Science 283: 1482-1488, 1999.

37. Reddy PH and Beal MF: Amyloid beta, mitochondrial dysfunction and synaptic damage: Implications for cognitive decline in aging and Alzheimer's disease. Trends Mol Med 14: 45-53, 2008.

38. Sultana R and Butterfield DA: Oxidatively modified, mitochondria-relevant brain proteins in subjects with Alzheimer disease and mild cognitive impairment. J Bioenerg Biomembr 41: 441-446, 2009.

39. Lu G, Liang R and Xie JQ: Characteristics of afferent fiber innervation on acupoint Zusanli (Chin). Sci Sin 22: 495-503, 1979.

40. Glenner GG and Wong CW: Alzheimer's disease: Initial report of the purification and characterization of a novel cerebrovascular amyloid protein. Biochem Biophys Res Commun 120: 885-890, 1984.

41. Tabner BJ, Turnbull S, El-Agnaf OM and Allsop D: Formation of hydrogen peroxide and hydroxyl radicals from $\mathrm{A}($ beta) and alpha-synuclein as a possible mechanism of cell death in Alzheimer's disease and Parkinson's disease 1,2. Free Radic Biol Med 32: 1076-1083, 2002

42. McBride HM, Neuspiel M and Wasiak S: Mitochondria: More than just a powerhouse. Curr Biol 16: R551-R560, 2006.

43. Humphries AD, Streimann IC, Stojanovski D, Johnston AJ, Yano M, Hoogenraad NJ and Ryan MT: Dissection of the mitochondrial import and assembly pathway for human Tom40. J Biol Chem 280: 11535-11543, 2005

44. Strittmatter WJ, Weisgraber KH, Huang DY, Dong LM, Salvesen GS, Pericak-Vance M, Schmechel D, Saunders AM, Goldgaber D and Roses AD: Binding of human apolipoprotein E to synthetic amyloid beta peptide: Isoform-specific effects and implications for late-onset Alzheimer disease. Proc Natl Acad Sci USA 90: 8098-8102, 1993.

45. Sanan DA, Weisgraber KH, Russell SJ, Mahley RW, Huang D, Saunders A, Schmechel D, Wisniewski T, Frangione B and Roses AD: Apolipoprotein E associates with beta amyloid peptide of Alzheimer's disease to form novel monofibrils. Isoform apoE4 associates more efficiently than apoE3. J Clin Invest 94: 860-869, 1994.

46. Ye S, Huang Y, Müllendorff K, Dong L, Giedt G, Meng EC, Cohen FE, Kuntz ID, Weisgraber KH and Mahley RW: Apolipoprotein (apo) E4 enhances amyloid $\beta$ peptide production in cultured neuronal cells: ApoE structure as a potential therapeutic target. Proc Natl Acad Sci USA 102: 18700-18705, 2005.

47. Salhab M, Patani N, Jiang W and Mokbel K: High TIMM17A expression is associated with adverse pathological and clinical outcomes in human breast cancer. Breast Cancer 19: 153-160, 2012.

This work is licensed under a Creative Commons Attribution-NonCommercial-NoDerivatives 4.0 International (CC BY-NC-ND 4.0) License. 